\title{
Reconstruction of Large Facial Defects via Excision of Skin Cancer Using Two or More Regional Flaps
}

\author{
Dong Min Lee ${ }^{1}$, Yong Chan Bae ${ }^{1,2}$, Su Bong Nam ${ }^{1}$, Seong Hwan Bae ${ }^{1}$, June Seok Choi ${ }^{1}$ \\ ${ }^{1}$ Department of Plastic and Reconstructive Surgery, Pusan National University School of Medicine, Busan; ${ }^{2}$ Biomedical Research Institute, \\ Pusan National University Hospital, Busan, Korea
}

Background Free-flap surgery and skin grafting can be used to reconstruct large facial defects that may result after skin cancer removal by Mohs micrographic surgery (MMS). However, these two methods may produce low postsurgical patient satisfaction with aesthetics, and free-flap surgery may produce an undue burden for the majority of skin cancer patients, who are of advanced age. Hence, the authors examined outcomes of large facial defect reconstruction using multiple flaps.

Methods Among patients undergoing MMS for skin cancer at Pusan National University Hospital between January 2013 and December 2015, we evaluated 7 patients (2 males, 5 females; average age, 73.14 years) treated for large facial defects from basal cell carcinoma. Based on operative and follow-up records, we investigated the number and type of flaps used, postsurgical complications, and patients' post-surgical satisfaction.

Results Two and 3 types of flaps were used for 5 and 2 patients, respectively. Most frequently used were nasolabial flaps ( 7 times in 6 patients) and forehead flaps (once in each of 4 patients). The average follow-up period was 14 months, with no complications-including necrosis, hematoma, or wound dehiscence-observed. Post-surgical satisfaction averaged 4.4 out of a maximum of 5 points.

Conclusions Reconstruction using two or more flaps for large facial defects after skin cancer removal using MMS produced satisfactory outcomes while preventing aesthetic problems. Practitioners should consider using multiple flaps when choosing a reconstruction method for large facial defects following skin cancer removal.

Keywords Mohs surgery / Multiple flaps / Large defects
Correspondence: Yong Chan Bae Department of Plastic and Reconstructive Surgery, Pusan National University School of Medicine; Biomedical Research Institute, Pusan National University Hospital, 179 Gudeok-ro, Seo-gu, Busan 49241, Korea

Tel: +82-51-240-7273

Fax: +82-51-243-9405

E-mail: baeyc2@hanmail.net

Received: 7 Nov 2016 • Revised: 16 Jun 2017 • Accepted: 27 Jun 2017

pISSN: 2234-6163 • elSSN: 2234-6171 • https://doi.org/10.5999/aps.2017.44.4.319 • Arch Plast Surg 2017;44:319-323

This article was presented as a poster at the 6th Research \&t Reconstructive Forum on Apr 7-8, 2016 in Seoul, Korea

No potential conflict of interest relevant to this article was reported.

\section{INTRODUCTION}

For a large defect region in which either primary closure or reconstruction using only one local flap cannot be done, it may be challenging to use only one kind of flap. If this is the case, a skin graft or free flap can be considered. Skin grafting is useful for covering large defect regions due to the fact that it seldom poses limitations to the range of reconstruction; however, its use is limited in cases where severe deformation is expected due to post-surgical constriction. A free flap is applicable even for cases with a deep or large defect region, or one with major structures exposed; however, free flaps result in low post-surgical satisfaction for patients in terms of aesthetics, and the potential burden of surgery should be considered, given that most skin cancer pa- 
tients are of advanced age.

Mohs micrographic surgery (MMS) is a suitable surgical technique for aesthetically sensitive sites such as the facial region when taking post-surgical reconstruction into account. It allows for the complete removal of cancer through real-time biopsy at the surgical margin while conducting excision, and at the same time, compared to wide excision, minimizes the loss of surrounding normal tissue. The most common application of MMS is the treatment of non-melanoma skin cancers. Particularly, randomized controlled trials conducted based on patients who had undergone basal cell carcinoma excision with MMS in the previous 10 years have reported a $4 \%$ recurrence [1].

Even with MMS, it may be impossible to avoid a large defect region while removing cancer. Hence, the authors conducted reconstruction in facial regions with large defects by using two or more flaps simultaneously, and analyzed the outcomes.

\section{METHODS}

This study evaluated 7 patients presenting a large defect region from among patients who underwent MMS after diagnosis with skin cancer at Pusan University Hospital during the period between January 2013 and December 2015. The average age of the patients was 73 years old, ranging between 68 and 79, and the patients included 2 males and 5 females. All of the patients had facial basal cell carcinoma. A follow-up study was done for all data based on surgical records and medical records during the follow-up period (Table 1).

The authors investigated the number of flaps used for each patient, the kind of flap used, the presence of post-surgical complications, and patients' post-surgical satisfaction. Patients' postsurgical satisfaction was evaluated through interviews with patients based on a 5 -point scale (1, very poor; 2 , poor; 3 , moderate; 4 , well; 5 , very well).

\section{RESULTS}

Regarding the kinds of flaps, various flaps including the median forehead flap, paramedian forehead flap, VY advancement flap, and transposition flap were used according to the judgment of the surgeon. Two kinds of flaps and 3 kinds of flaps were used simultaneously for 5 patients and 2 patients, respectively, for a total of 7 patients with multiple flaps. Nasolabial flaps and forehead flaps were used with high frequency; the former were used 7 times in 6 patients, while the latter were used 4 times in 4 patients. The average period of post-surgical follow-up observation for patients was 14 months, and no complications - including necrosis, hematoma, or wound dehiscence - were observed. Patients' post-surgical satisfaction was evaluated on a 5-point scale. Almost all reported a 5, two patients a 4, and the other one a 3 (Table 1).

The patient in the first case we discuss in depth (Case no. 5 in Table 1) was a 68-year-old male who complained of a pigmented plaque lesion on the left side of the nose that had been present since a year before the visit, and he was diagnosed with basal cell carcinoma from a biopsy. MMS was conducted under local anesthesia in our clinic, the tumor negative margin was confirmed, and the area of the defect region at the time was 45 $\mathrm{mm} \times 28 \mathrm{~mm}$. The defect involved exposed cartilage due to the removal of the full thickness of the alar skin. The defect was too small and shallow for a free flap, and a skin graft was considered to be unsuitable for aesthetic reasons, due to its location in the exact center of the face and its deep depth. Coverage with only one local flap was determined to be impossible and reconstruction was conducted by using two local flaps, including a bilobed flap from the nasal dorsum side and a transposition flap from the left nasolabial region. The patient is under follow-up observation in an outpatient clinic, and there have been no abnormal findings, including recurrence, flap necrosis, or hematoma, in the 9 months since the reconstruction (Fig. 1).

Table 1. The characteristics of patients, the flaps used for each patient, and patient satisfaction

\begin{tabular}{|c|c|c|c|c|}
\hline $\begin{array}{l}\text { Case } \\
\text { no. }\end{array}$ & Sex/Age (yr) & Diagnosis \& location & Flaps used for reconstruction & Satisfaction \\
\hline 1 & Female/70 & Basal cell carcinoma \& nasal alar region (left) & Median forehead flap and nasolabial advancement flap & 4 \\
\hline 2 & Female/71 & Basal cell carcinoma \& upper lip (left) & Nasolabial transposition flap and vermilion advancement flap & 5 \\
\hline 3 & Female/76 & Basal cell carcinoma \& nasal side wall (left) & $\begin{array}{l}\text { Median forehead flap, advancement flap on cheek and } \\
\text { nasolabial V-Y advancement flap }\end{array}$ & 5 \\
\hline 4 & Female/79 & Basal cell carcinoma \& nasal alar and nasolabial fold region (right) & Median forehead flap and nasolabial V-Y advancement flap & 5 \\
\hline 5 & Male/68 & Basal cell carcinoma \& nasal alar region (left) & Bilobed flap and nasolabial transposition flap & 5 \\
\hline 6 & Female/76 & Basal cell carcinoma \& nasal tip & $\begin{array}{l}\text { Paramedian forehead flap, nasolabial transposition flap (right), } \\
\text { and nasolabial rotation advancement flap (left) }\end{array}$ & 3 \\
\hline 7 & Male/72 & Basal cell carcinoma \& nasolabial fold region (right) & $\begin{array}{l}\text { Advancement flap on cheek and musculocutaneous flap } \\
\text { advancement on upper lip }\end{array}$ & 4 \\
\hline
\end{tabular}




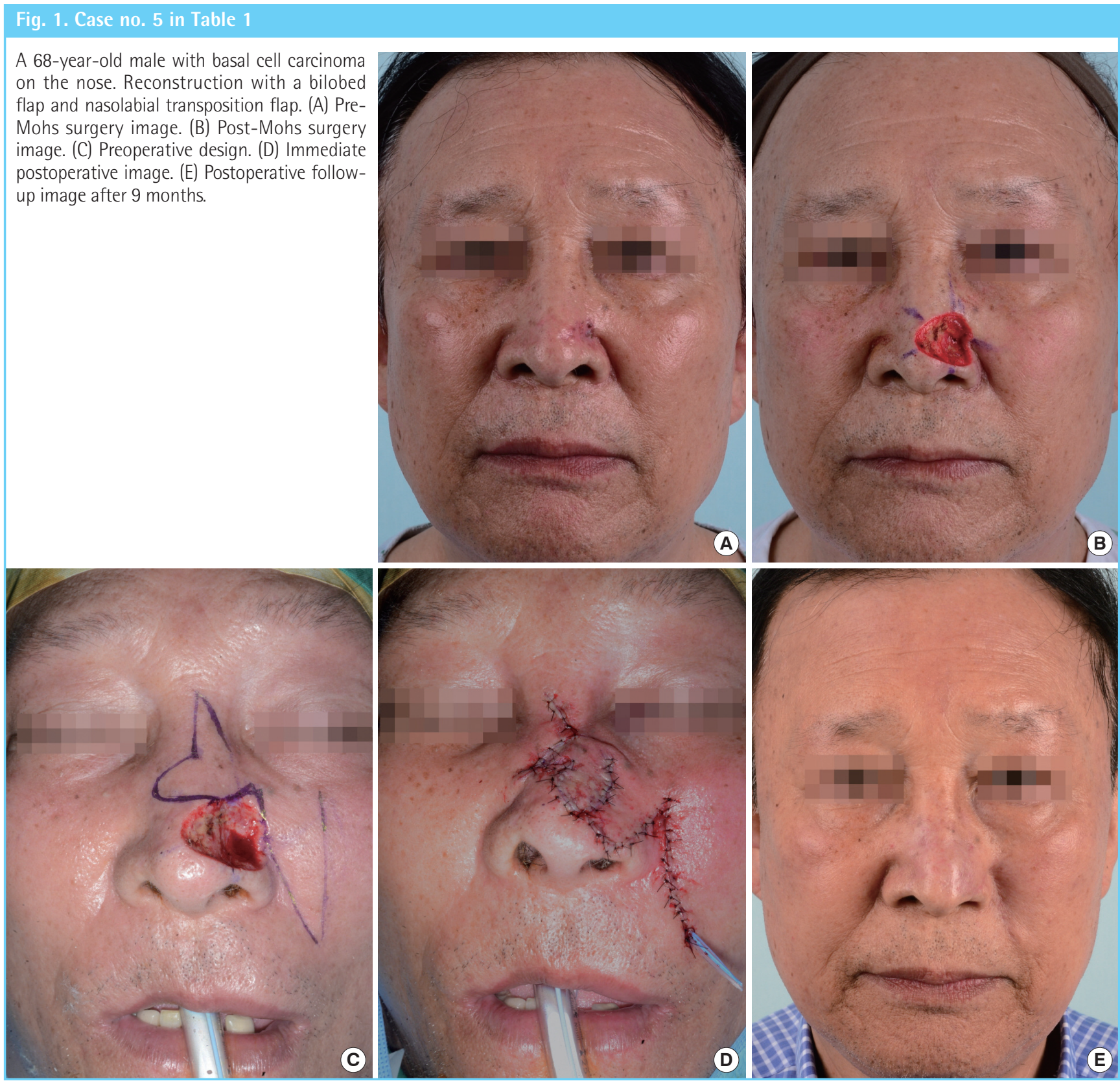

The patient in the second case we discuss in depth (Case no. 6 in Table 1) was a 76-year-old female who reported a nodule on the left side of the nose that had been present for 10 years before the visit. She was diagnosed with basal cell carcinoma from a biopsy conducted due to bleeding for 2 years. Like the patient in Case 5 discussed above, a full-thickness defect in the alar skin was observed, and the cartilage was exposed. A free flap was ruled out when considering post-surgical aesthetic satisfaction and the patient's advanced age, regardless of the large size of the defect. A skin graft was also predicted to result in low satisfaction with the aesthetics of the treatment owing to the depth of the defect; hence, reconstruction with various local flaps was considered. Consequently, reconstruction with a paramedian forehead flap, a nasolabial transposition (left), and a nasolabial rotation advancement flap (right) was conducted, and flap division and insetting for the forehead flap were conducted 3 weeks after reconstruction with the flap. The last picture is the photograph taken 6 months after conducting flap division. The patient is under follow-up observation in the outpatient clinic and like all the other patients in this study, no other abnormal findings have been observed, including recurrence, flap necrosis, or hematoma, up to 8 months after the reconstruction (Fig. 2). 


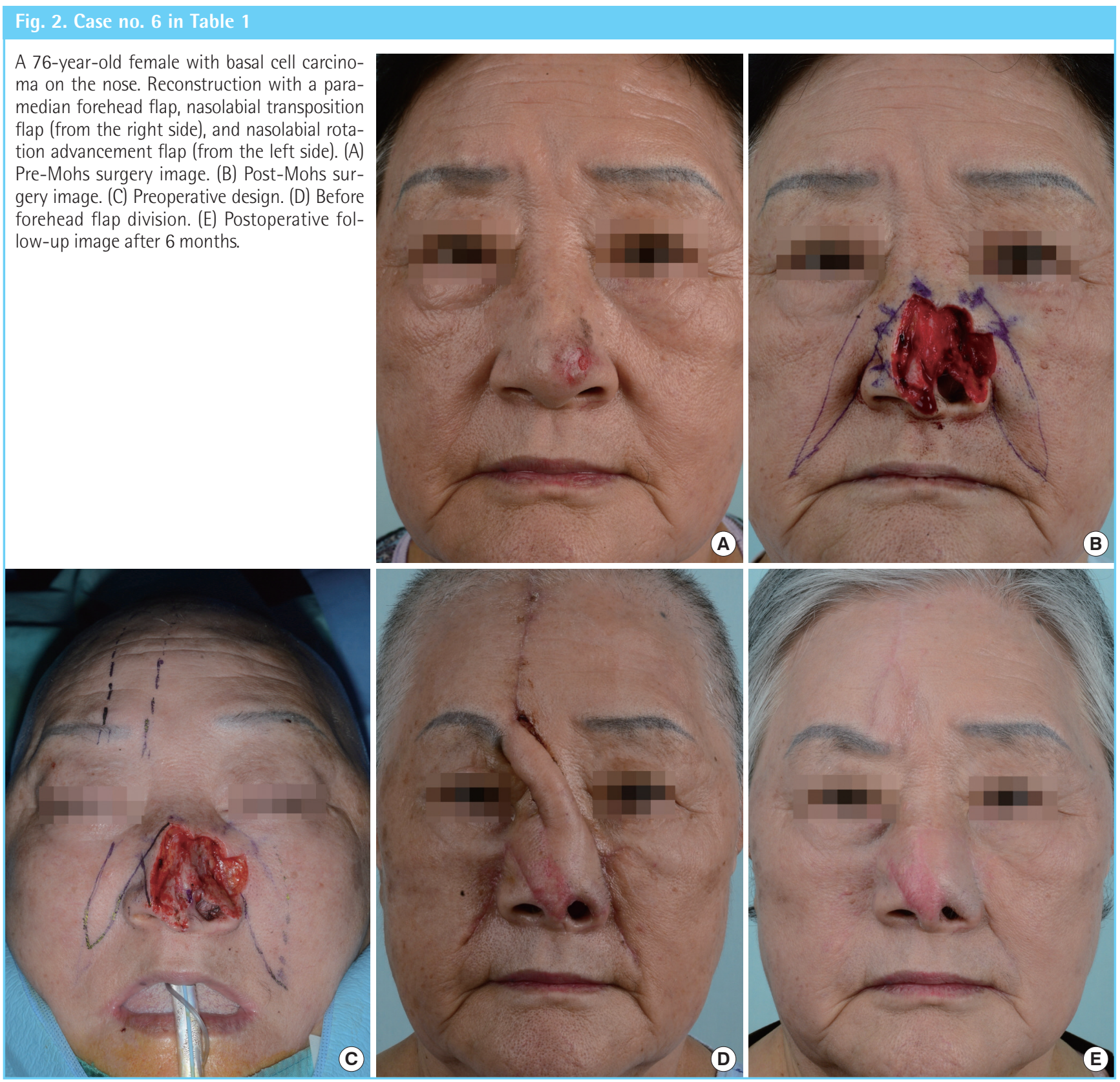

\section{DISCUSSION}

MMS allows for the complete removal of cancer while minimizing the loss of normal tissue surrounding the tumor in aesthetically sensitive regions such as the face [1].

However, most cases are challenging for reconstruction using only one simple local flap because of the size of the defect region resulting from removal with MMS, owing to the dimensions of the tumor. In such cases, a skin graft or free flap can be considered. However, skin grafts result in a typical 'patch' appearance due to color and contour differences from the surrounding tissue and, thereby, patients' aesthetic satisfaction is low compared to a flap. In the case of a free flap for skin cancer, required in patients who are mostly of advanced age, factors to take into consideration include the general condition of the patient who is to undergo significant surgery with a long operation time, the patient's capacity for recovery after the operation, and the duration of hospitalization. Measures to take if the free flap fails must be prepared for. As with a skin graft, aesthetic issues also must be considered.

Suarez et al. [2] reported a successful case of reconstruction using a bilateral nasolabial VY advancement flap and a VY glabella advancement flap for a large defect extending from the nasal dorsum to the bilateral medial canthus. In addition, Wollina 
[3] confirmed poor aesthetic outcomes for patients who received a skin graft when compared with a flap in his study, in which various cases of reconstruction methods for large defects in the facial region resulting from MMS were considered. Among Wollina's 19 cases of reconstruction for a large defect, multiple local flaps were used in 1 case, a combination of a local flap and skin graft was used in 1 case, solely a skin graft was used in 2 cases, and the remaining 15 cases were treated with a simple local flap. However, in the case of East Asian patients, a local flap is difficult to apply to large defects due to differences in skin elasticity; and the use of multiple local flaps is essential.

Another issue of note is that the skin cancer for all patients in this study existed either on the nose or the region near the nose. The external nose is one of the most vulnerable sites for skin cancer and is the site with the most complicated structure for reconstruction [4]. There are numerous methods for reconstruction of the nose, and each method has its own weaknesses and strengths [5]. Among these methods, a forehead flap has been recognized for its safety and its aesthetically and functionally excellent outcomes by many researchers, regardless of the inconvenience imposed by this 2-stage procedure [6,7]. A nasolabial fold can be a useful perforator flap for perinasal reconstruction, as it carries a reliable and constant cutaneous perforator from the facial artery [8]. The authors frequently use such a surgical technique; however, there are limitations when using only one method for a large defect, as shown in this study. This also indicates the necessity of using multiple local flaps, as mentioned above.

As we can surmise from the phrase "the next nearest skin is the best skin" from Gillies and Millard in the 1950s [2], reconstruction of a defect region using flaps in the cosmetically sensitive facial region definitively yields satisfactory aesthetic outcomes, as undamaged skin is used that is the most similar in color tone, pigmentation, elasticity, texture, and skin thickness to the skin of the defect region [4]. Hence, the authors have tried to achieve satisfactory aesthetic outcomes while reducing the burden on patients by using two or more flaps.

For a large defect in the facial region caused after the removal of a lesion through MMS in skin cancer patients, the authors of this study conducted reconstruction using two or more flaps si- multaneously for cases where reconstruction with only one flap was difficult. Ultimately, for skin cancer patients who are mostly of advanced age, surgery that may be a burden, such as free flap surgery, was avoided, and sufficiently satisfactory outcomes were achieved while preventing the aesthetic issues that tend to occur with skin grafts.

Hence, practitioners should choose a method for reconstruction after considering simultaneous use of two or more flaps for a large defect following the removal of skin cancer in the facial region.

\section{REFERENCES}

1. Thomas CL, Lam A, Lam J, et al. Factors affecting choice of repair in Mohs micrographic surgery for non-melanoma skin cancer of the head. Australas J Dermatol 2016 Feb 24 [Epub]. https:// doi.org/10.1111/ajd.12453.

2. Suarez JE, Sadigh PL, Jeng SF. Aesthetic subunit reconstruction facilitated with $\mathrm{v}-\mathrm{y}$ island advancement flaps on the face: a case report. Plast Reconstr Surg Glob Open 2014;2:e148.

3. Wollina U. Reconstruction of large facial defects after delayed Mohs surgery for skin cancer. Acta Dermatovenerol Croat 2015;23:265-9.

4. Deluca J, Tappeiner L, Pichler M, et al. Using the Peng flap for a wide dorsal nasal defect. J Dtsch Dermatol Ges 2014; 12:1060-2.

5. Lindsay KJ, Morton JD. Flap or graft: The best of both in nasal ala reconstruction. J Plast Reconstr Aesthet Surg 2015; 68:1352-7.

6. Stigall LE, Bramlette TB, Zitelli JA, et al. The paramidline forehead flap: a clinical and microanatomic study. Dermatol Surg 2016;42:764-71.

7. Blazquez-Sanchez N, Fernandez-Canedo I, Repiso-Jimenez $\mathrm{JB}$, et al. Usefulness of the paramedian forehead flap in nasal reconstructive surgery: a retrospective series of 41 patients. Actas Dermosifiliogr 2016;107:133-41.

8. Camuzard O, Foissac R, Georgiou C, et al. Facial artery perforator flap for reconstruction of perinasal defects: an anatomical study and clinical application. J Craniomaxillofac Surg 2015;43:2057-65. 